\title{
Design of Advanced Static Var Generator
}

\author{
Na Zheng ${ }^{a}$, Qinghua Bai ${ }^{b}$ \\ School of Electrical and Information Engineering, Beihua University, Jilin 132021, China \\ azhengna1981@163.com, bbaichuanshui@163.com
}

Keywords: Advanced Static Var Generator, Digital Signal Processor, reactive power compensation.

\begin{abstract}
This template explains the working principle of reactive power compensation of ASVG firstly, selects reactive current control mode to compensate reactive power, and uses composite switch to control the compensation device switching and off. In hardware circuit, it uses TMS320LF2407 DSP chip as the core controller to complete the sampling signal, A/D conversion, data processing, control, real time calculation and other functions, which to realize the fast response of dynamic compensation of power system; the main circuit of ASVG and the voltage and the current signal detecting, collecting and adjust circuit are designed.
\end{abstract}

\section{Introduction}

The development of reactive power compensation device in power system started from the capacitor, went through synchronous condenser, static var compensation device and ASVG (Advanced Static Var Generator) several different stages. The effect of capacitor, synchronous condenser and the static var compensation device for compensating is not very well, ASVG as a reactive power regulation device, has become the development direction of modern reactive power compensation device, become the key of research topic for domestic and abroad power system industry. This paper describes the design of a small capacity reactive power compensation device can be widely used in small power load side. The device compensates on the spot, improves the power factor of the load, and can improve the power supply quality and economic benefit of power grid.

\section{Principle of ASVG}

Static var generator mainly refers to using of three-phase bridge converter circuit through the reactor connected in parallel to the grid, through adjusting the AC side output voltage amplitude and phase of inverted bridge circuit, or direct control the AC side current, to generate or absorb reactive power to meet the requirements, thus realizing full range of dynamic compensation from inductive to capacitive reactive power.

Take the single-phase equivalent circuit to explain the principle of ASVG, the equivalent circuit shown in figure 1.

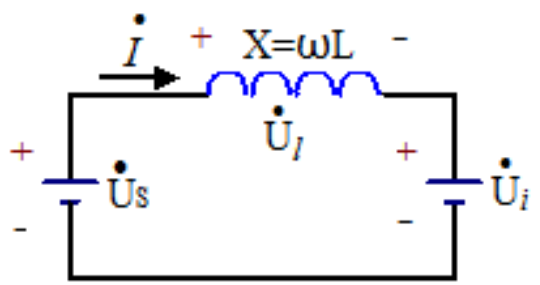

Fig. 1 Equivalent circuit of ASVG device

It assumes the grid voltage and the AC voltage of ASVG output as $\dot{U}_{s}$ and $\dot{U}_{i}$, so the voltage $\dot{U}_{l}$ of reactance $\mathrm{X}$ is the phasor dissimilarity of $\dot{U}_{s}$ and $\dot{U}_{i}$, while the current of connection reactance can be controlled by the voltage. Therefore, changing the amplitude of the ASVG AC side output voltage $\dot{U}_{i}$ and phase relative to the system voltage $\dot{U}_{s}$, it can change the voltage connection 
reactance, so as to control the phase and the amplitude of the ASVG current absorbing from the power grid, will control the ASVG properties and size of absorption reactive power. When $\dot{U}_{i}$ is larger than $\dot{U}_{s}$, the current is ahead of system voltage $90^{\circ}$, ASVG absorbs from capacitive reactive power ; when less than, the current delays system voltage $90^{\circ}$ and absorbs from inductive reactive power.

\section{Switch of Reactive power compensation device}

The switch is the most important part of the reactive power compensation device, which controls the switching operations of compensation device group, because of its frequent switching, which lead to the most easily damaged. This design uses a novel switch -- composite switch. Composite switch is mainly comprised of control board, thyristor and magnetic latching relay [1], the principle block diagram is shown in figure 2.

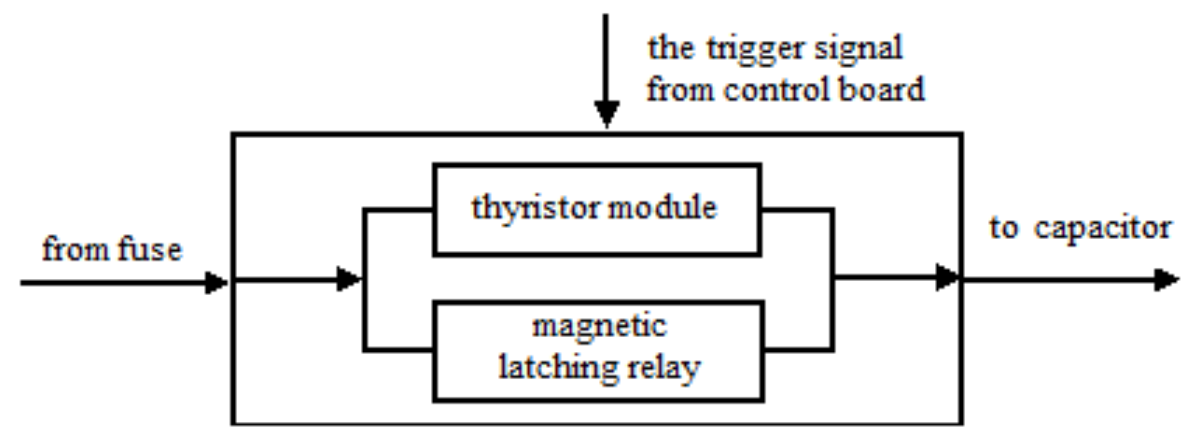

Fig.2 Principle diagram of compound switch module

The process of composite switch input compensation device: distribution integrated controller sends a trigger signal to the composite switch, after combination switch control board receiving the signal, starts to detect the zero crossing point of the voltage and connects to the main loop when the voltage zero is crossing by the thyristors, magnetic latching relay after a certain delay, pull in the potential case. Because the thyristor is a contactless switch, and zero potential investment, after working, the relay contact resistance is far less than the thyristor resistance, the current through the magnetic latching relay contact into the power grid. Therefore, avoids switching compensation device of inrush current, arc, ignition, reduces the thyristor loss.

The process of composite switch removal compensation device: after the controller output trigger signal disappearing, the magnetic latching relay disconnect in equipotential case, thyristor by delayed, at current zero off. This switch is fully taken advantage of contactless switch thyristor and relay, therefore composite switch is ideal for switching compensation device.

\section{Design of control system hardware circuit}

The system design of the hardware of control system. The hardware structure diagram of reactive power compensation device control system is shown in Figure 3, mainly includes a main controller module, voltage and current detection module, pulse drive circuit module, the compound switch module and communication module, memory module, display module etc..

The design of the main circuit of ASVG. The main circuit structure of ASVG device is shown in Figure 4. One of the key technologies of ASVG is to ensure that the AC side of converter can output high quality, amplitude and phase adjustable AC voltage. Therefore, this paper adopts full controlled power device IGBT which is the high pressure, high switching frequency, the low loss of switch [2]. The DC side parallel connect with a large capacity capacitor, as inverter, converter through the reactor in parallel to the electric network, which constitute the main circuit of ASVG device. 


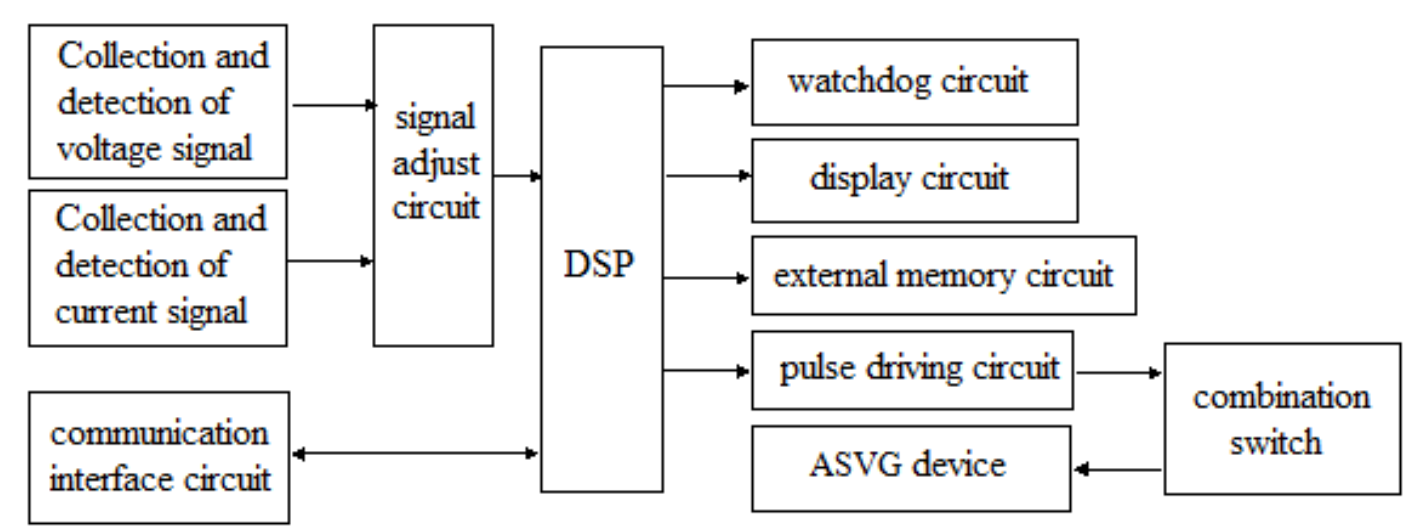

Fig.3 Hardware structure diagram of control system

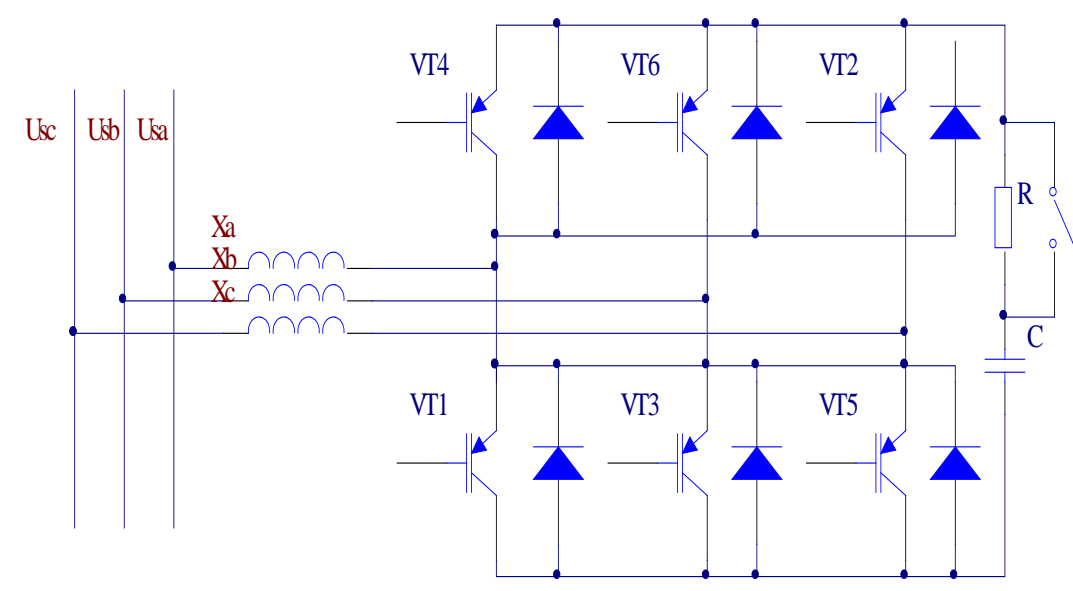

Fig.4 The structure diagram of ASVG device main circuit

Module design of core controller. The controller is adopted TMS320LF2407A DSP chip, and completed that data processing and calculation, the whole system control signaling, and external communication etc. [3] Out of the DSP, It is directly connected to a 64k16bit, the working voltage +3.3V SRAM-CY7C1021, 32K space for the expansion of the data memory, The rest of 32K for the expansion of the program memory. Communication interface is used RS-232 to realize the transmission of local data.

The circuit design of voltage, current signal detection, acquisition and adjust. When signal detecting, it will be transform strong signals into weak small signal by the Holzer sensor firstly. The A phase voltage from three phase circuit is input to the circuit in Figure 5, the A phase current is input to the circuit in Figure 6, the circuit of the B, C two-phase is similar.

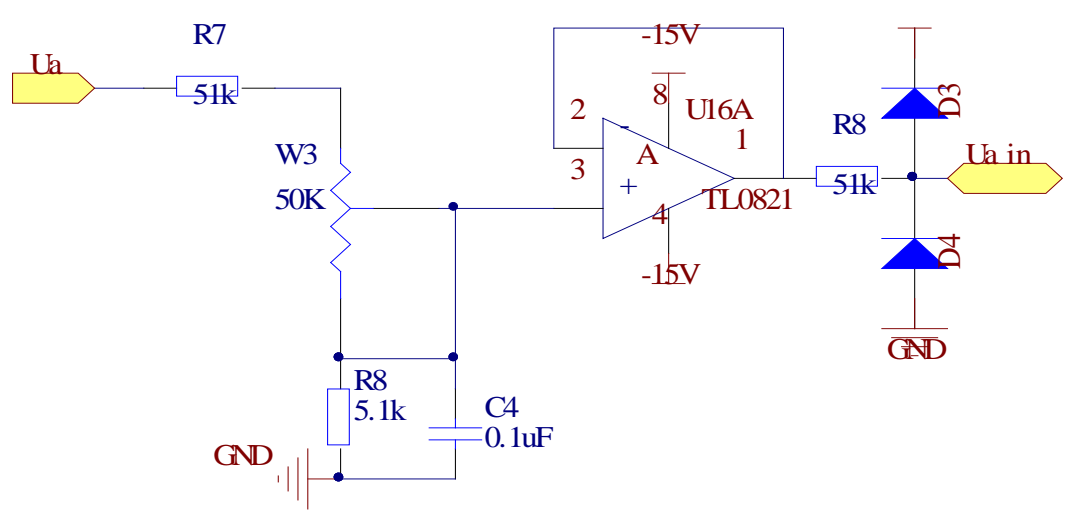

Fig. 5 The detection circuit of the A phase voltage signal 


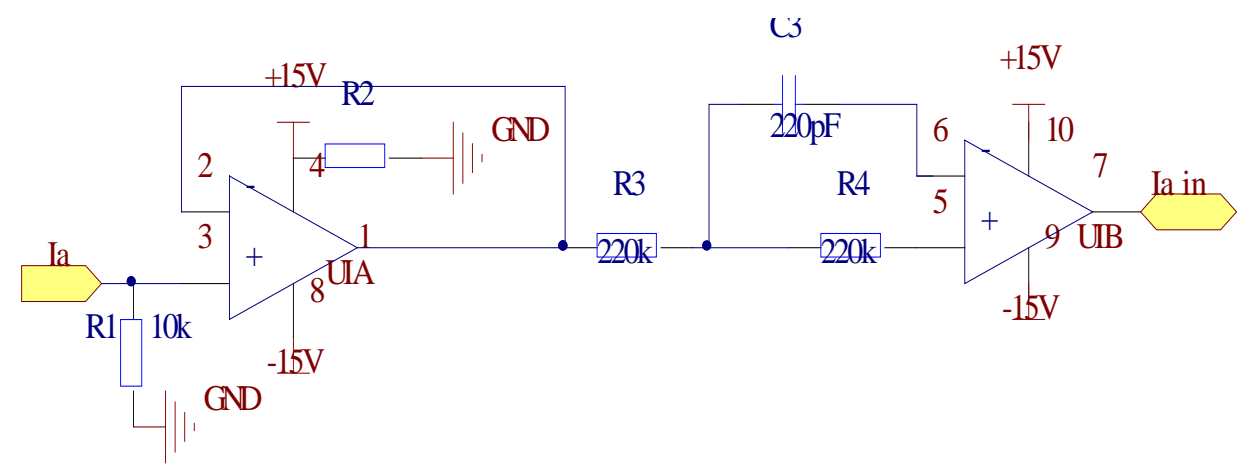

Fig.6 The detection circuit of the A phase circuit signal

The signals which detected by the voltage, current conversion circuit is sent to the AC sampling circuit cannot directly use, it is necessary to design a signal adjust circuit makes signal can meet the requirement [4]. After adjusting, the signals which converted by the A/D become that DSP can identify and processing.

\section{Conclusion}

Advanced static var generator device is designed in this paper using high frequency and fully controlled power electronic devices IGBT in the main circuit, control circuit is using DSP as control core, to ensure high precision and fast calculation results. Using compound switch, it is a good technical foundation for the development of intelligent reactive power compensation.

\section{References}

[1] Menggang Wei, Peng Li, Yande He.Power Electronics,Research of composite switch for reactive power compensation capacitor,2013(8).

[2] Xuekui Wang,Xuhong Yang,Jianhua Ye. The research of IGBT driver module.Journal of Shanghai University of Electric Power. 2008(3)

[3] Haipeng Li, Peifeng Niu. Development of ASVG controller based on DSP, Relay, 2005(10)

[4] Wenjiang Li; Shanshan Li; Gaihua Wang, Design of signal adjust circuit of the static var generator, Measurement \& Control Technology, 2014(7) 